\title{
MS09-P01 | IMPROVING DENSITY HISTOGRAM BY PHASE OPTIMISATION USING A GENETIC
}

\section{ALGORITHM}

Kantamneni, Sravya Mounika (European Molecular Biology Laboratory, Hamburg, GER); Sobolev, Egor (European Molecular Biology Laboratory, Hamburg, GER); Lamzin, Victor S (European Molecular Biology Laboratory, Hamburg, GER)

In macromolecular crystallography the initial phases obtained by experimental phasing or molecular replacement may not always be sufficiently accurate to produce an interpretable density map. Additional phase improvement steps using density modification and/or model refinement approaches may be required. Given the complexity of the phase space to be sampled, heuristic global optimisation techniques based on genetic algorithms may have their own advantages.

A sampling of the phase space and phase optimisation using genetic algorithms has been attempted and has produced promising results for reflections at low resolution of the data and for the use of the third moment of density distribution, skewness, as a target function [1, 2].

Here we present a phase optimisation approach using a genetic algorithm with several characteristics of the density map as a target function. For two selected test cases with X-ray data to $2.5 \AA$ resolution, we observe the development of the density histogram with a gradual shift towards the histogram of the map computed from the refined and deposited model.

Keywords: Electron density, Skewness, Genetic algorithm

[1] Lunin et al., (2016) Acta Cryst. D72, 147-157.

[2] Uervirojnangkoorn et al., (2013) Acta Cryst. D69, 2039-2049. 